\title{
Prevalência de automedicação em profissionais de saúde de um hospital privado de São Luís-MA
}

\author{
Prevalence of self medication for health professionals of a private hospital São Luis-MA
}

\author{
Wellison Amorim Pereira1, José Augusto Rodrigues de Almeida², Raíssa Guará Assunção", \\ Elizângela Araújo Pestana Motta ${ }^{4}$.
}

\begin{abstract}
Resumo. Introdução: A automedicação tem sido objeto de muitas pesquisas e assume uma importância maior quando é realizada por profissionais de saúde, principalmente pelo acesso aos medicamentos. Objetivo: Considerando a automedicação como um problema de saúde pública e a importância de conhecer a ocorrência desta prática por aqueles profissionais, este estudo objetivou avaliar a prevalência da automedicação e os fatores associados em profissionais de saúde de um hospital privado em São Luís, Maranhão. Metodologia: Caracteriza-se por um estudo transversal, com coleta de dados por meio de questionário autoaplicável, com perguntas abertas e fechadas, no período de fevereiro a abril de 2015. Resultados: Dos 120 profissionais entrevistados, 73,3\% $(n=88)$ faziam uso da automedicação, sendo $80 \%$ ( $n=96)$ mulheres; $45 \%(n=54)$ tem ensino médio completo, 36,7\% $(n=44)$ possuem renda de até 3 salários, $33,3 \%(n=40)$ técnicos de enfermagem e $47,5 \%(n=57)$ trabalhavam em dois turnos. Os principais problemas de saúde apontados para a automedicação foram a cefaléia $42 \%(n=37)$, infecções ou inflamações $36,4 \%$ $(n=32)$ e gripe ou resfriado $35,2 \%(n=31)$. Conclusão: Esta pesquisa aponta para um elevado consumo de medicamentos entre profissionais de saúde, principalmente no ambiente hospitalar. Ressalta-se aqui a figura do farmacêutico, a fim de elucidar dúvidas quanto ao tratamento, visando o uso racional e correto de medicamentos.
\end{abstract}

Palavras-chave: automedicação; uso de medicamentos; profissionais de saúde.

Abstract. Introduction: Self-medication has been the object of much research and is of greater importance when it is performed by health professionals, especially for access to medicines. Objective: Considering selfmedication as a public health problem and the importance of knowing the occurrence of this practice by those professionals, this study aimed to evaluate the prevalence of self-medication and associated factors in health professionals of a private hospital in São Luís, Maranhão. Methodology: Characterized by a cross-sectional study, with data collection through a self-administered questionnaire, with open and closed questions, from February to April 2015. Results: Of the 120 professionals interviewed, $73.3 \%(n=88)$ used self-medication, with $80 \%(n=96)$ women; 45\% $(n=54)$ had a high school education, 36.7\% $(n=44)$ had incomes of up to 3 salaries, $33.3 \%(n=40)$ nursing technicians and $47.5 \%$ worked two shifts. The main health problems reported for self-care were headache, $42 \%(n=37)$, infections or inflammations $(n=32)$ and flu or cold $35.4 \%(n=31)$. Conclusion: This research points to a high consumption of drugs among health professionals, especially in the hospital environment. The figure of the pharmacist is emphasized here, in order to elucidate doubts regarding the treatment, aiming at the rational and correct use of medicines.

Keywords: self-medication; drug utilization; health professionals.

\footnotetext{
${ }^{1}$ Mestrando (a) em Biologia Parasitária. UNICEUMA

${ }^{2}$ Farmacêutico. Instituto Florence

${ }^{3}$ Doutoranda em Ciências da Saúde (UFMA). Membro do CEP da UNICEUMA.
}

Autor de correspondência: email: elifarmabr@gmail.com

Endereço: Rua Rio Branco, 216 - Centro, São Luís - MA, 65020-470.

Telefone: (98) 98413-8428

Rev. Investig, Bioméd. São Luís, 10(2): 142-154, 2018 


\section{Introdução}

Os medicamentos tais como hoje os conhecemos são relativamente recentes. No começo do século XIX a maioria dos medicamentos era remédios de origem natural, de estrutura química e natureza desconhecidas ${ }^{1}$.

Nos últimos anos ocorreu a introdução maciça de novos fármacos, que trouxeram à população possibilidade de cura para enfermidades até então fatais, sobretudo no campo de doenças infecciosas. Os avanços nas pesquisas de novos fármacos, em conjunto com sua promoção comercial, criaram uma excessiva crença da sociedade em relação ao poder dos medicamentos. A prescrição torna-se quase obrigatória nas consultas médicas, sendo este fato, sinônimo de boa prática médica, justificando sua enorme demanda 2 .

A despeito dos inegáveis ganhos terapêuticos obtidos, os medicamentos passaram a sofrer um uso indiscriminado e irracional ${ }^{3}$. Para o uso racional de medicamentos, é preciso, em primeiro lugar, estabelecer a necessidade do medicamento, que se receite o medicamento apropriado de acordo com os ditames de eficácia e segurança comprovados. Além disso, é necessário que o medicamento seja prescrito adequadamente, na forma farmacêutica, doses e período de duração do tratamento; que esteja disponível a um preço acessível, e que responda sempre aos critérios de qualidade exigidos, com a necessária orientação e responsabilidade, e, finalmente, que se cumpra o regime terapêutico já prescrito, da melhor maneira possível ${ }^{4}$.

Em países desenvolvidos, o número de medicamentos de venda livre tem crescido, assim como a disponibilidade desses medicamentos em estabelecimentos não farmacêuticos, o que favorece 0 processo de automedicação. Nesses países, no entanto, os rígidos controles estabelecidos pelas agências reguladoras e 0 crescente envolvimento dos farmacêuticos com a orientação dos usuários de medicamentos, tornam menos problemática a prática do autocuidado. Já no Brasil, onde o acesso à assistência médica pública é difícil e onde há uma grande parcela da sociedade na faixa da pobreza que não tem condições financeiras para pagar um plano de saúde, a prática da automedicação torna-se bastante comum..$^{5,6}$..

Os profissionais de saúde constituem um grupo de trabalhadores vulneráveis à prática do auto-cuidado, devido à interação entre habilidades técnicas (compreensão do processo saúde-doença), relações interpessoais e crescentes responsabilidades ${ }^{7}$. Loyola Filho, et $\mathrm{al}^{8}$ (2002) observou uma prevalência de $24,8 \%$ da automedicação entre estes profissionais; já em um estudo feito no Estado do Rio Grande do Sul, foi observada prevalência mais elevada, representando 53,3\% dos trabalhadores da área ${ }^{9}$. Entre os profissionais adeptos dessa prática destacam-se médicos, enfermeiros e odontólogos ${ }^{10}$, sendo que a prevalência em enfermeiros era de $32 \%$, inferior apenas à prevalência identificada entre os médicos $(43 \%)^{11}$. Em alguns estudos a prática da automedicação foi observada com uma maior prevalência nos enfermeiros quando comparados aos auxiliares e técnicos ${ }^{12}$.

Os farmacêuticos são os profissionais de saúde responsáveis pela automedicação responsável, cuja formação inclui disciplinas que abordam não só a composição, mas também os efeitos farmacológicos e deletérios dos medicamentos ${ }^{13}$. O autocuidado orientado pelo farmacêutico é 
visto atualmente como uma realidade irreversível e já é considerado como parte integrante dos sistemas de saúde. Ele permite uma maior autonomia por parte da população nos cuidados com sua própria saúde e colabora com os governos na medida em que evita um número insustentável de consultas médicas ${ }^{14}$. É notável a inclusão do farmacêutico no processo de automedicação responsável e, torna-se imprescindível para o mesmo ter a noção exata de sua competência e dos limites de sua intervenção no processo saúde-doença, para que assuma a atitude correta, no momento oportuno, avaliando a situação do doente, conduzindo-o, se necessário, a uma consulta médica ou ao hospital, em caso de urgência ${ }^{13,15}$.

A necessidade do conhecimento por parte da comunidade científica e da sociedade sobre a realidade por trás da automedicação em profissionais da área da saúde foi fator motivador para a realização desta pesquisa, uma vez que em saúde pública um dos aspectos de menos estudo na avaliação da qualidade da atenção está relacionado aos cuidadores, ou seja, àqueles profissionais que são responsáveis pelo atendimento direto dos usuários de serviços de saúde. Considerando os riscos que a automedicação pode oferecer na saúde pública e a importância de se conhecer as implicações resultantes dessa problemática para a sociedade, este estudo tem como objetivo caracterizar a prevalência da automedicação em profissionais de saúde de um hospital privado em São Luís - MA.

\section{Material e métodos}

Foi realizado um estudo transversal, com abordagem descritiva e quantitativa em um hospital da rede privada em São Luís, Maranhão, entre os meses de fevereiro a abril de 2015 . Foram convidados a participar da pesquisa profissional da saúde de ensino superior e técnico, atuantes no hospital e maiores de 18. A amostra total foi de 120 profissionais, selecionados aleatoriamente, dos turnos diurno e noturno, a saber: enfermeiros, técnicos de enfermagem, técnicos em radiologia, nutricionistas, técnicos em nutrição, fisioterapeutas, farmacêuticos, técnicos de laboratório e técnicos em farmácia. Excluiu-se da amostragem o médico, por este ter autonomia para se medicar.

Os profissionais de saúde foram consultados sobre a participação voluntária na pesquisa e aqueles que aceitaram participar assinaram o Termo de Consentimento Livre e Esclarecido (TCLE) e preencheram um questionário autoaplicável e não identificável, formulado com 15 questões abertas e fechadas. Os participantes foram informados quanto à finalidade do estudo, sobre o sigilo quanto à identidade e sobre 0 direito de suspender a participação a qualquer momento. $O$ questionário foi preenchido pelos participantes no turno de trabalho em que os mesmos se encontravam. O questionário foi entregue ao profissional, que optava em responder no ato da entrega pelo pesquisador ou entregar no mesmo dia, após algum tempo.

O questionário continha duas partes: na primeira consistia de dados relacionados à caracterização dos trabalhadores como variáveis sóciodemográficas (sexo, escolaridade, estado civil, se tem plano de saúde) e dados profissionais (profissão, turno em que trabalha e se realiza ou não plantão noturno). Na segunda parte havia questões sobre a prática da automedicação, se fazia ou não uso, motivo do uso, quem indicou 0 medicamento, problema de saúde tratado pela automedicação, 
conhecimento dos usuários quanto aos riscos de se automedicarem e reação adversa decorrente do uso. Ao final foi perguntado se o ambiente de trabalho influenciava na decisão do uso do medicamento.

Os dados foram digitados no Microsoft Exce/( 2013 e analisados no Biostat versão 5.3. Foram realizadas análises descritivas, médias e desvio padrão e aplicado o teste Qui-quadrado com o coeficiente de variação de 95\% e nível de significância de 5\%. Esta pesquisa foi aprovada pelo Comitê de Ética em Pesquisa Plataforma Brasil, sob 0 protocolo $\mathrm{n}^{-}$ 43167715.0.0000.5084.

\section{Resultados e discussão}

Dos 120 participantes da pesquisa em relação à primeira parte do questionário, a maioria corresponde ao sexo feminino com $80 \%(n=96)$. E do total entrevistado apenas $5,8 \%(n=7)$ cursaram somente 0 ensino fundamental, $45 \%$ ( $n=54)$ concluíram o ensino médio e apenas $20,8 \% \quad(n=25)$ possuíam nível superior. A profissão de maior participação na pesquisa foi a de técnico em enfermagem com 33,3\% $(n=40)$, seguida de enfermeiros $16,7 \%$ $(n=20)$. Observou-se que houve uma prevalência tanto para o trabalho no turno diurno $(49,2 \%)$ como para dois turnos $(45,8 \%)$. A Tabela 1 apresenta a prevalência do uso de fármacos conforme características sociodemográficas e profissionais dos participantes do estudo.

O estudo revelou que $80 \%$ da amostra $(n=96)$ é formada por mulheres, resultado semelhante ao encontrado por Barros e colaboradores $(86,6 \%)^{14}(2009)$, onde $70,8 \% \quad(n=68)$ destas relataram se automedicar, e dentre os 24 homens, $83,3 \% \quad(n=20)$ relataram o mesmo. A avaliação da relação entre o gênero da amostra e a automedicação ficou prejudicada devido à disparidade da amostragem, uma vez que os profissionais para o qual a pesquisa foi aplicada são na sua maioria do sexo feminino. Todavia, a maioria dos estudos evidencia que a prática da automedicação é maior nas mulheres, contudo não é consensual ${ }^{17}$. Bush \& Osterweis ${ }^{16}$ atribuem esse achado, entre outras razões, a mais frequente utilização de serviços de saúde pelas mulheres. Já para Loyola Filho et $\mathrm{al}^{17}$, o maior consumo de medicamentos prescritos entre as mulheres pode estar associado à maior presença de condições crônicas e queixas e à maior utilização de serviços de saúde.

Em geral, o consumo de medicamentos costuma aumentar à medida que as pessoas envelhecem ${ }^{18,19}$. No entanto, a utilização de automedicação tende a ser maior entre os mais jovens ${ }^{15,20}$. De forma semelhante aos resultados deste estudo, indivíduos com maior grau de instrução tendem a se automedicar mais ${ }^{21-24}$. $O$ aumento da automedicação entre pessoas com maior escolaridade já havia sido relatado por Loyola Filho ${ }^{8}$ e Vilarino ${ }^{40}$. O conhecimento geral e experiências de vida tornam o indivíduo mais confiante para se automedicar" 40 . As razões para isso têm sido atribuídas a fatores tais como: maior conhecimento sobre os medicamentos, maior poder econômico, menor confiança nos médicos e maior sentimento de autonomia pessoal diante de decisões sobre a própria saúde ${ }^{20}$.

Sobre a utilização de medicamentos sem prescrição nos últimos seis meses, $73,3 \% \quad(n=88)$ estavam utilizando e apenas $26,7 \%$ $(n=32)$ afirmaram não estar fazendo uso (Figura 1). O uso de medicamentos sem prescrição médica é um hábito muito frequente na população brasileira ${ }^{25}$. Tal fato pode ser observa- 
Tabela 1: Prevalência de automedicação segundo variáveis sociodemográficas e profissionais de trabalhadores da saúde de hospital privado.

\begin{tabular}{|c|c|c|c|}
\hline VARIÁVEL & $\mathbf{N}$ & PERCENTUAL (\%) & PREVALÊNCIA (\%) \\
\hline \multicolumn{4}{|l|}{ SEXO } \\
\hline Masculino & 24 & 20 & 83,3 \\
\hline Feminino & 96 & 80 & 70,8 \\
\hline \multicolumn{4}{|l|}{ ESTADO CIVIL } \\
\hline Casado & 52 & 43,3 & 67,3 \\
\hline Solteiro & 52 & 43,3 & 73,1 \\
\hline Viúvo & 2 & 1,7 & 100 \\
\hline Amigado & 11 & 9,2 & 90,9 \\
\hline Divorciado / separado & 3 & 2,5 & 100 \\
\hline ESCOLARIDADE & & & \\
\hline Ensino fundamental completo & 7 & 5,8 & 85,7 \\
\hline Ensino médio completo & 54 & 45 & 70,4 \\
\hline Ensino médio incompleto & 2 & 1,7 & 50 \\
\hline Ensino superior completo & 25 & 20,8 & 92 \\
\hline Ensino superior incompleto & 9 & 7,5 & 77,8 \\
\hline Especialização & 21 & 17,5 & 57,1 \\
\hline Mestrado & 2 & 1,7 & 50 \\
\hline \multicolumn{4}{|l|}{ RENDA FAMILIAR } \\
\hline$\leq 2$ salários & 40 & 33,3 & 75 \\
\hline 2 a 3 salários & 44 & 36,7 & 77,3 \\
\hline $\begin{array}{l}4 \text { ou mais } \\
\text { CARGO OU FUNÇÃO }\end{array}$ & 36 & 30 & 66,7 \\
\hline Enfermeiro & 20 & 16,7 & 75 \\
\hline Técnico de enfermagem & 40 & 33,4 & 77,5 \\
\hline Farmacêutico & 10 & 8,3 & 100 \\
\hline Técnico de laboratório & 10 & 8,3 & 100 \\
\hline Fisioterapeuta & 10 & 8,3 & 50 \\
\hline Nutricionista & 4 & 3,4 & 25 \\
\hline Técnico em nutrição & 6 & 5 & 83,3 \\
\hline Técnico em farmácia & 10 & 8,3 & 40 \\
\hline $\begin{array}{l}\text { Técnico em radiologia } \\
\text { PLANO DE SAÚDE }\end{array}$ & 10 & 8,3 & 70 \\
\hline Possui & 51 & 42,5 & 70,6 \\
\hline $\begin{array}{l}\text { Não possui } \\
\text { TURNO DE TRABALHO }\end{array}$ & 69 & 57,5 & 75,4 \\
\hline Diurno & 59 & 49,2 & 72,9 \\
\hline Noturno & 6 & 5,0 & 83,3 \\
\hline Dois turnos & 55 & 45,8 & 72,7 \\
\hline
\end{tabular}


do em uma pesquisa encomendada pelo laboratório Bayer ao Instituto Marplan, a qual mostrou que $49 \%$ das mulheres entrevistadas em oito capitais brasileiras não saem de casa sem um analgésico a tiracolo ${ }^{25}$. Outra pesquisa, realizada pelo Departamento de Psicobiologia da Universidade Federal de São Paulo (Unifesp) em 2001, mostrou que cerca de $22 \%$ da população toma comprimidos para facilitar o sono e mais da metade (62\%) não passou por consulta médica ${ }^{25}$. É possível fazer uma análise a este resultado segundo um estudo realizado por Lopes ${ }^{26}$, em que 91,3\% dos entrevistados admitiram recorrer à automedicação quando apresentam problemas de saúde.

Figura 1. Distribuição percentual dos dados referente ao consumo de medicamentos sem prescrição nos últimos seis meses em trabalhadores da saúde de hospital.

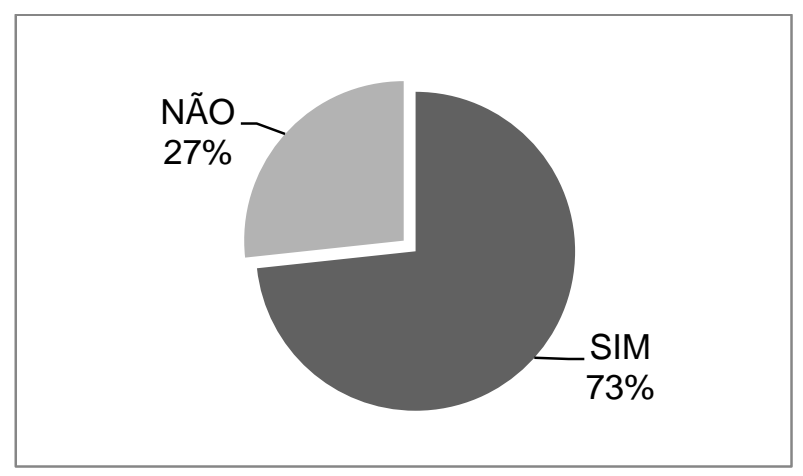

A prevalência de automedicação referida entre os profissionais de saúde avaliados foi de 73,3\%, um número muito alto se comparado a outros estudos como o relatado por Loyola Filho ${ }^{8}$ em Minas Gerais, realizado com moradores acima de 18 anos, onde verificou uma prevalência de $24,8 \%$. Essa proporção foi superior àquela identificada entre os profissionais da rede básica de saúde da zona urbana de Pelotas-RS $(32,4 \%)^{7}$. As frequências foram também superiores à prevalência descrita por Villarino e colaboradores $^{39}$ em estudo com residentes do município de Santa Maria, Rio Grande do Sul, que descrevem uma prevalência de $53,3 \%{ }^{14}$. Por outro lado, foi mais alta do que a observada na população adulta de outros países, tais como a Espanha $(12,7 \%)^{7}$.

Mesmo diante do resultado já esperado e do baixo nível de evidência dos artigos sobre o tema, é possível inferir que a prevalência da automedicação encontrada neste estudo se assemelha à população em geral. Conforme estudo realizado em 12 capitais, 76,4\% dos brasileiros praticam a automedicação. Entre as capitais pesquisadas foram encontradas as seguintes prevalências: Salvador/BA (96,2\%); Recife/CE (96\%); Manaus/AM (92\%); Rio de Janeiro RJ $(91,4 \%) ; \quad$ Brasília DF $(83,1 \%) ;$ São Paulo/SP (83\%); Belém/PA (78\%); Curitiba/PR (66\%); Goiânia/GO (65\%); Fortaleza/CE (53\%); Porto Alegre/RS (49\%) e Belo Horizonte/MG $(35 \%)^{27}$ (2014).

Ao responderem sobre quem indica o uso de medicamento: 47,8\% ( $n=42)$ profissional de saúde, $25 \%(n=22)$ através de prescrição anterior (antiga), 14,8\% $(n=13)$ familiar ou amigo, 6,8\% $\quad(n=6)$ colega de trabalho e $4,5 \% \quad(n=4)$ balconistas, conforme apresentado na Figura 2. Verificou-se que entre os diferentes meios de automedicação, o que assumiu maior expressão neste estudo é o recurso a medicamentos indicados por profissional de saúde $(47,8 \%)$, o que configura a prática da automedicação com controle de algum profissional de saúde. Segundo Santos ${ }^{28}$, as pessoas não hesitam, quando se trata de aliviar as suas dores passageiras, em tomar medicamentos por sugestões de familiares, amigos ou rendendo-se ao impulso da publicidade. Estas pessoas não acreditam que os sintomas de malestar são sempre individuais e que qualquer medicamento deve ter uma utilização personalizada. A afirmação deste autor poderá ser uma possível explicação que faz com que ainda $52,2 \%$ dos indivíduos entrevistados se 
automediquem sem orientação de algum profissional de saúde.

Infelizmente, muitos medicamentos são usados sem prescrição médica e quem toma geralmente recorre a um balconista, familiares, pessoas mais velhas. Em pesquisa realizada em Vassouras, Rio de Janeiro ${ }^{29}$ em 2004, ao serem questionados a respeito dos critérios utilizados para a escolha de tais medicamentos, $25,86 \%(n=30)$ receberam indicação de parentes; 14,7\% $(n=17)$ utilizaram receita antiga para adquirir o medicamento utilizado anteriormente para tratar o mesmo sintoma, 19,0\% $(n=22)$ receberam indicação de balconistas em farmácias e drogarias da região, 11,2\% $(n=13)$ escolheram após terem visto a propaganda do medicamento, 4,31\% $(n=5)$ receberam indicação do farmacêutico e $25 \%(n=29)$ utilizaram por conta própria sem precisar do motivo exato da escolha do medicamento.

Com relação aos motivos de recorrer à automedicação: 42\% (n=37) disseram ser um problema de saúde simples, 35,2\% $(n=31)$ sabem qual a doença e o medicamento indicado e $19,3 \% \quad(n=17)$ argumentaram a falta de tempo para ir ao médico. Conforme a Figura 3, verificou-se que a maior parte dos indivíduos consideram ter sintomas comuns e irrelevantes que não necessitam de consulta médica para serem resolvidos, ou então, quando tentam consultar o médico encontram problemas do sistema de saúde como o tempo de espera ou a falta de vagas, mesmo no sistema privado, que fazem com que os indivíduos recorram à automedicação para assim restabelecer mais rapidamente a sua saúde.

Figura 2. Distribuição percentual dos dados segundo o agente influenciador da automedicação em trabalhadores da saúde de hospital privado.

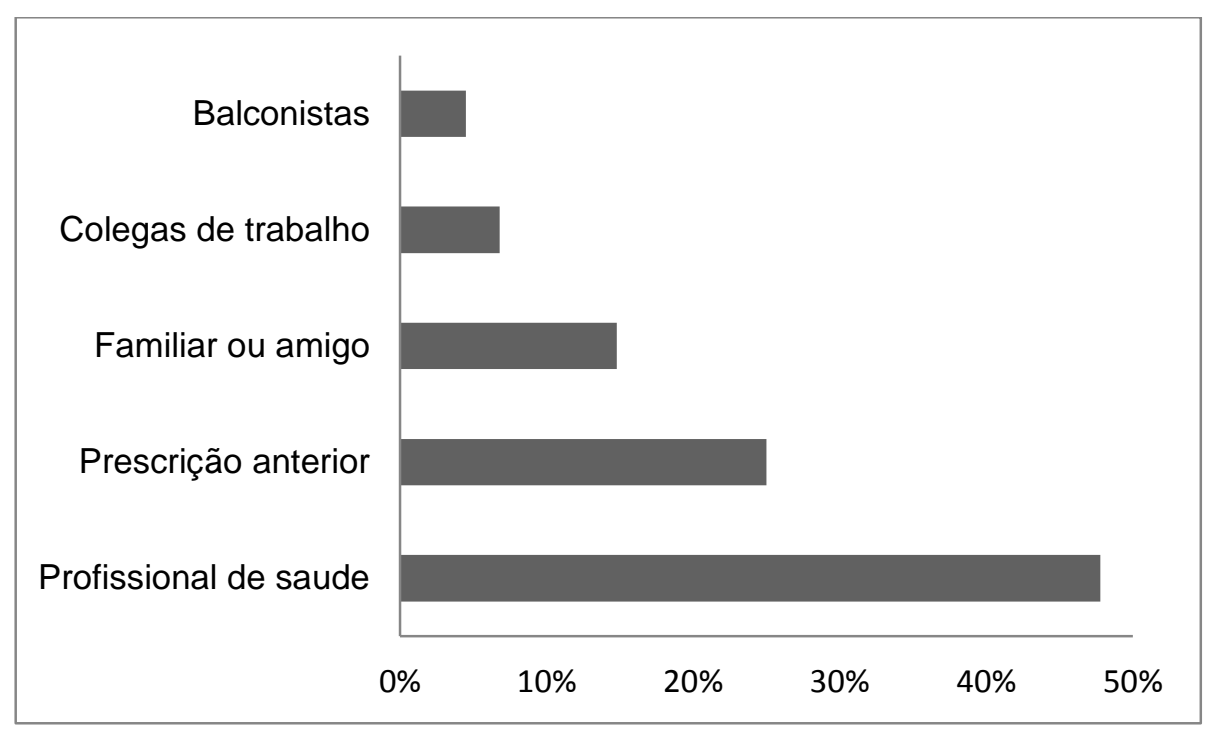


Figura 3. Distribuição percentual dos dados segundo os motivos que levaram a automedicação em trabalhadores da saúde de hospital privado.

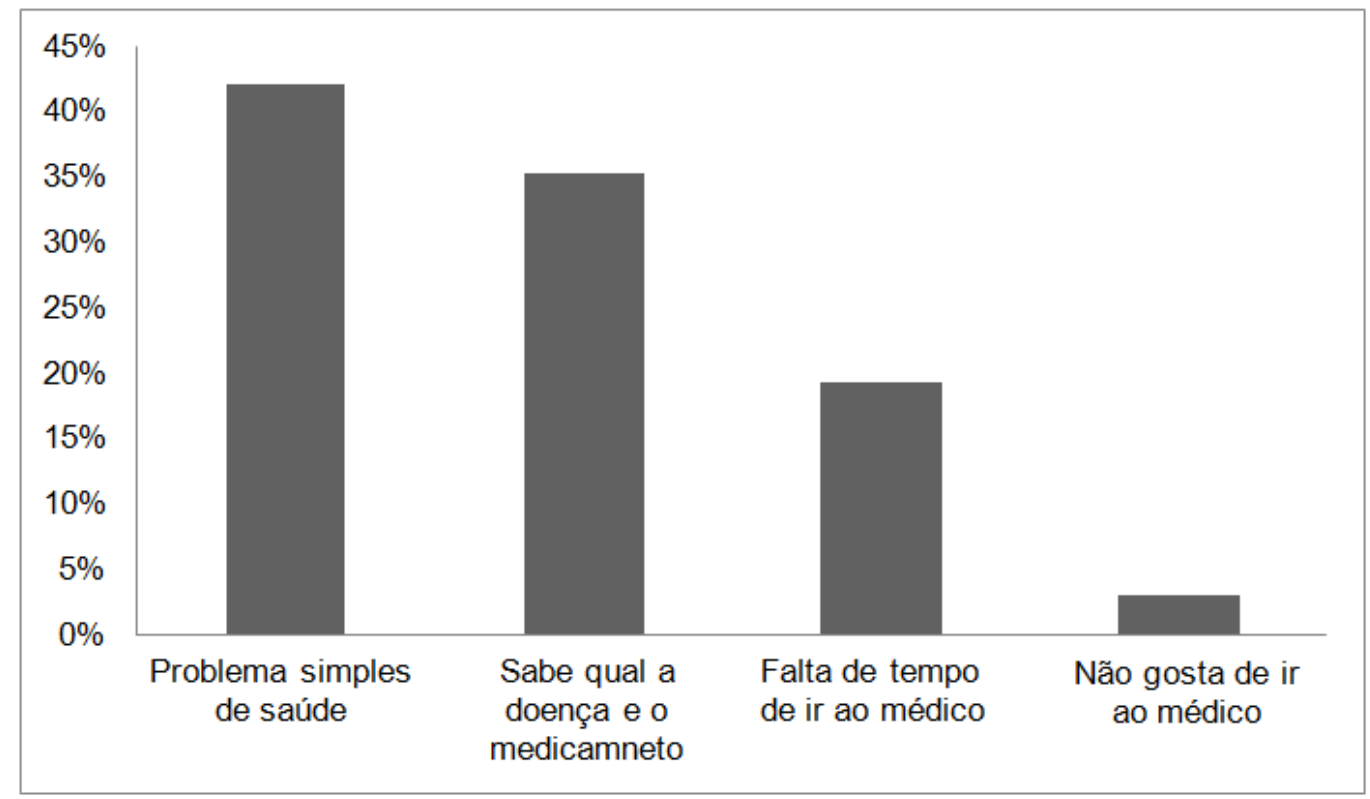

Estudos mostram a probabilidade maior de consultar um médico em indivíduos que trabalham menos de 40 horas semanais, pois dispondo de maior tempo livre, este trabalhador tem maior oportunidade de cuidar da própria saúde $^{30}$. O profissional tem consciência que a busca por atendimento especializado é importante, porém se refugia na falta de tempo para manter-se automedicando. Segundo Paulo \& Zanine $^{31}$, a automedicação é uma prática comum e atemporal; em especial hoje, graças a dificuldades de acesso a serviços de saúde. Sobre as doenças mais comuns que levam a automedicação: 42\% relataram cefaleia (dor de cabeça), infecções ou inflamações $(36,4 \%)$, gripe ou resfriado $(35,2 \%)$, dores na coluna $(20,4 \%)$ e febre $(15,9 \%)$, conforme Figura 4. Outros problemas, no entanto, foram citados, como cólicas, alergias, dor no estômago, manchas no corpo, tosse, labirintite e gastrite.

As dores de cabeça batem recorde no que se refere ao uso de medicamentos.
Constantemente as pessoas fazem uso de algum tipo de analgésico para combater esse mal. Verifica-se que existem certos sintomas que são considerados como menores e por isso não necessitam de consulta médica, ou nunca são referidos ao médico. Afigura-se neste âmbito que as questões de saúde que não alcançam o status de verdadeiro problema de saúde e que, por isso entram no domínio da automedicação, podem ser prejudiciais à saúde do indivíduo e pode nunca chegar a ser qualificado como verdadeiro problema de saúde justificativo de recurso a cuidados médicos.

Com relação aos riscos que o medicamento pode causar quase todos os indivíduos que participaram desta pesquisa e que se automedicaram tinham conhecimento desses riscos $-89,8 \%$ da amostra $(n=79)$ e somente $10,2 \% \quad(n=9)$ desconheciam esses riscos, conforme Figura 5. 
Figura 4. Distribuição percentual segundo os problemas mais comuns que levaram a automedicação em trabalhadores da saúde de hospital privado. Obs: *os profissionais para esse quesito poderiam marcar mais de uma opção.

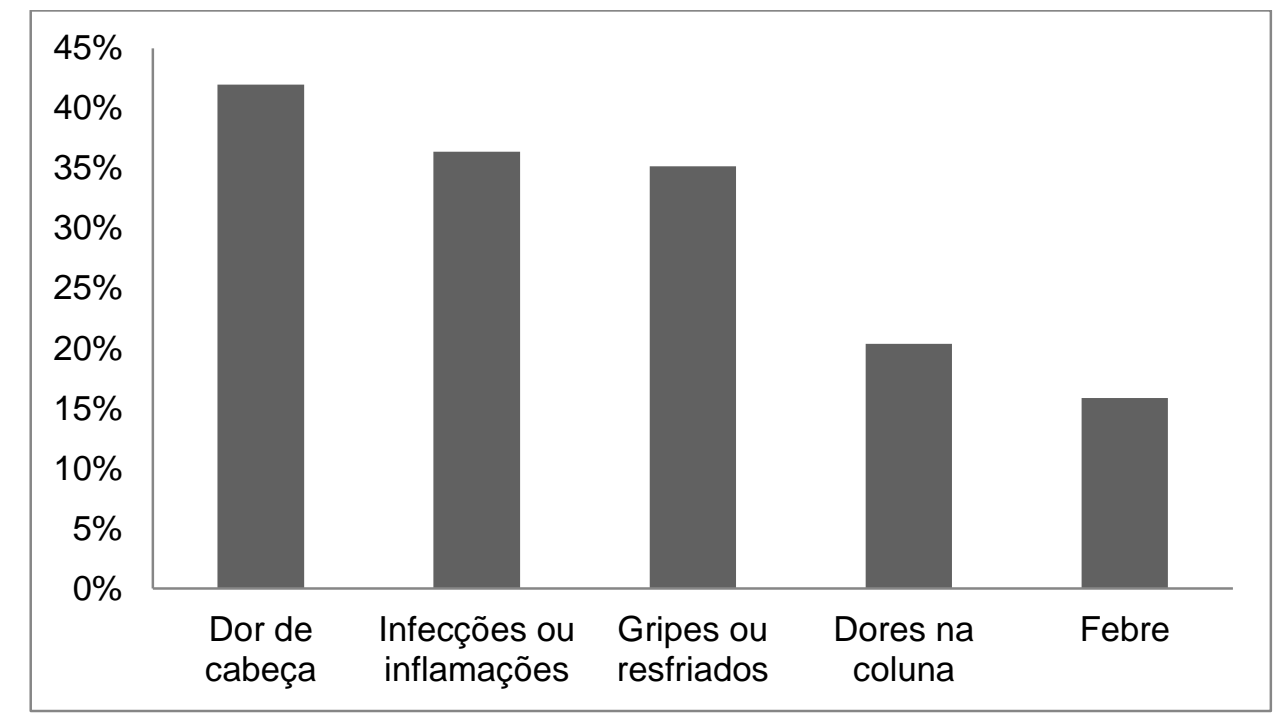

Figura 5. Distribuição percentual dos dados segundo o conhecimento dos possíveis riscos decorrentes da automedicação em trabalhadores da saúde de hospital privado.

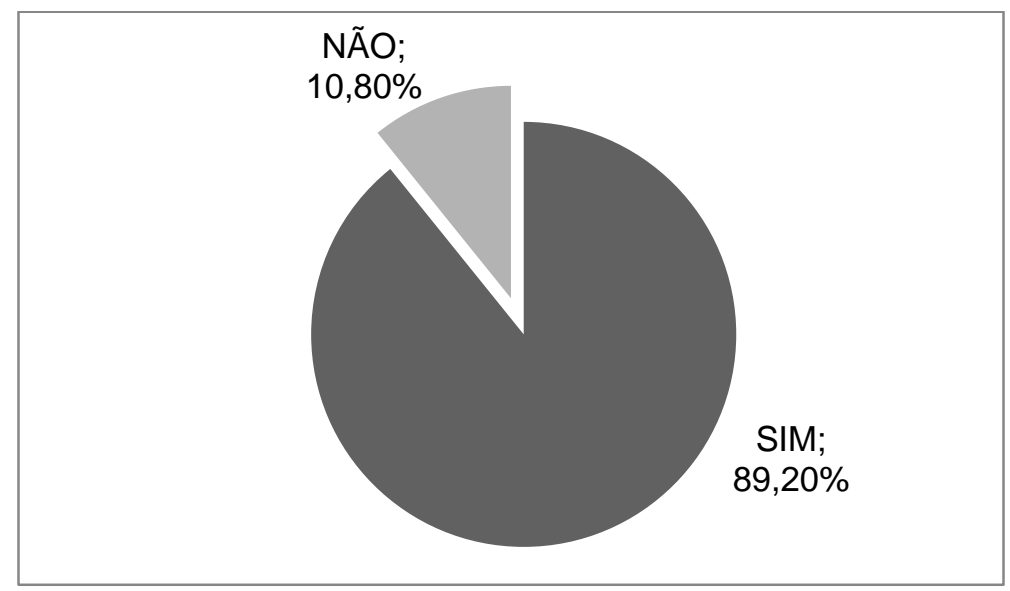

Deste modo pode-se afirmar que a automedicação praticada por estes indivíduos, de certa forma, representa um risco diminuído para a sua saúde. Porém foi verificado em estudo realizado com adultos que quase $100 \%$ destes referiram não ter conhecimento dos riscos que a automedicação podia causar ${ }^{32}$. Segundo Marques ${ }^{33}$ os incidentes relacionados com automedicação devem-se à negligência de quem a pratica, e refere ainda que estes incidentes medicamentosos poderiam ser evitados em $50 \%$ dos casos, se houvesse monitorização adequada.

A automedicação racional pode trazer benefícios para a saúde. Segundo a $\mathrm{OMS}^{34}$, a automedicação responsável é entendida como parte das ações de autocuidado. A OMS define autocuidado como o que as pessoas fazem por si mesmas para estabelecer e manter a saúde, prevenir e lidar com a doença.

Para Marques ${ }^{33}$, o risco que a automedicação pode causar está 
relacionado com a falta de informação que o indivíduo tem em relação aos efeitos indesejáveis e reações adversas que o medicamento pode causar, mas não é apenas a função do medicamento que causa esses riscos, mas também das características de quem o vai tomar e da correta adequação do perfil de indicações do medicamento à situação a tratar.

Em relação a problemas com a automedicação, é possível verificar que $8 \%(n=7)$ dos indivíduos admite ter tido e $92 \% \quad(n=81)$ refere não ter ocorrido nenhum problema. Embora não seja um número elevado de profissionais a ter este tipo de problemas, 7 indivíduos é um número significativo quando se verifica que o seu estado de saúde se agravou, onde 4 destes referem ter tido vômitos, diarreia e náuseas, 2 apresentaram dor no estômago e sonolência e por fim, 1 referiu ter tido reação alérgica ao medicamento. De um modo geral verifica-se que existe um número elevado de indivíduos que recorre à automedicação, e grande parte julga que os sintomas são comuns e ligeiros e que não justificam uma consulta médica. O seu comportamento frente à automedicação, embora apresente alguns cuidados, na sua maioria desenrola-se como uma automedicação não responsável. E assim sendo numa amostra de 120 indivíduos foi possível identificar 7 casos de problemas de saúde causados pela má prática da automedicação.

Sobre a influência do ambiente de trabalho na automedicação mais da metade afirmou que não tiveram influência do setor de trabalho na hora de se automedicar, todavia, houve um número significativo que relatou que tiveram, sim, influencia desse meio no autocuidado. Esse fato ocorre, principalmente, devido a três fatores: problemas de saúde adquiridos no ambiente de trabalho, acesso facilitado aos medicamentos indicados para esses problemas e possibilidade de "tratar" esses problemas de saúde de forma imediata e sem gastos $^{35}$.

A grande maioria dos profissionais de saúde entrevistados trabalha em regime de escala, 12 horas diurnas ou noturnas, às vezes, dobram o plantão, ficando expostos a situações que podem comprometer seu viver saudável em função do labor - tornando-se mais suscetíveis às doenças, a depressões e ao cansaço. Muitas vezes eles recorrem à automedicação para solucionar seus problemas de saúde.

$\mathrm{O}$ acesso aos medicamentos nos próprios serviços de saúde potencializa seu uso de forma abusiva. Martins ${ }^{33}$, em seu trabalho, relata que a facilidade de acesso do trabalhador de enfermagem, no caso das substâncias psicoativas, surge nas falas dos entrevistados. O processo de trabalho permite que estes lidem diretamente com os medicamentos e, quando percebem algum tipo de necessidade, visualizam solução relativamente imediata, sem gastos e de fácil acesso, sendo mais um fator de risco ao uso e abuso dos fármacos.

Com base no valor observado na Tabela 2, verificou-se que não existe diferença significativa em relação à categoria profissional e a automedicação, ou seja, todos praticam o autocuidado, apesar de a categoria farmacêutica ter apresentado uma prevalência de 100\%. Um estudo na Índia mostrou que a automedicação foi prevalente entre $67 \%$ dos farmacêuticos ${ }^{36}$. Em outro estudo que avaliou somente a automedicação com medicamentos não controlados entre farmacêuticos americanos, a maioria dos entrevistados (77\%) relatou automedicação com esses fármacos, e os demais $(31 \%)$ disseram nunca ter recebido tratamento médico para a doença ${ }^{37}$. 
Tabela 2: Prevalência de automedicação segundo correlação da profissão com o autocuidado em trabalhadores da saúde de hospital privado de São Luís - MA, 2015.

\begin{tabular}{lccccc}
\hline \multicolumn{1}{c}{ Profissão } & Sim & $\%$ & Não & $\%$ & Total \\
\hline Enfermeiro & 15 & 75 & 05 & 25 & 20 \\
Farmacêutico & 10 & 100 & - & - & 10 \\
Nutricionista & 01 & 25 & 03 & 75 & 04 \\
Fisioterapeuta & 05 & 50 & 05 & 50 & 10 \\
Tec. Enfermagem & 31 & 77,5 & 09 & 22,5 & 40 \\
Tec. Laboratório & 10 & 100 & - & - & 10 \\
Tec. Nutrição & 05 & 83,3 & 01 & 16,7 & 06 \\
Tec. Farmácia & 04 & 40 & 06 & 60 & 10 \\
Tec. Radiologia & 07 & 70 & 03 & 30 & 10 \\
Total & 88 & & 32 & & 120 \\
\hline
\end{tabular}

Os profissionais da enfermagem também apresentaram prevalências elevadas de automedicação, e conforme a literatura é a categoria de trabalhadores que mais se automedica. No presente estudo foram encontradas frequências altas do consumo de automedicação entre esses profissionais, até mesmo por estes serem a maioria da amostra. Observou-se que $77,5 \%$ dos auxiliares e técnicos de enfermagem e $75 \%$ dos enfermeiros utilizavam de modo frequente ou esporádico de medicações sem recomendação médica. Barros e colaboradores relataram em seu estudo a prevalência de automedicação em enfermeiros quando comparados com todo o grupo de enfermagem, assemelhando-se também com 0 resultado de um estudo realizado na Suíça em profissionais de nível superior em medicina no qual foi encontrada maior incidência da automedicação nesses profissionais $^{14,38}$.

\section{Conclusão}

A causa mais comum de automedicação entre profissionais da saúde é a cefaleia $e$ as dores osteomusculares; já os principais motivos que levam estes profissionais a se automedicar é a autoconfiança, a facilidade de acesso aos fármacos, a dificuldade de acesso aos serviços de saúde (enquanto usuários) e pela falta de tempo. Tal prática é mais comum até a quarta década de vida e entre os que possuem nível de escolaridade elevado. Assim, os dados apresentados revelam a necessidade de um trabalho de conscientização desses profissionais. 


\section{Referências}

1. Vosgerau MZS. et al. Consumo de medicamentos entre adultos na área de abrangência de uma Unidade de Saúde da Família. Ciencia \& saude coletiva. 2011; 16 : 1629-1638.

2. Vosgerau MZS. et al. Consumo de medicamentos entre adultos na área de abrangência de uma Unidade de Saúde da Família. Ciencia \& saude coletiva. 2011; 16 : 1629-1638.

3. Bonfilio $R$. et al. Farmácia magistral: sua importância e seu perfil de qualidade. Revista Baiana de Saúde Pública. 2010; 653-664.

4. Sant'ana JMB. et al. Racionalidade terapêutica: elementos médico-sanitários nas demandas judiciais de medicamentos. Revista de Saúde Pública. 2011; 45: 714-721.

5. Marin E. et al. Avaliação da automedicação com antiinflamatórios não-esteróides em farmácias comerciais de Santa MariaRS. Disciplinarum Scientia| Saúde. 2016; 6.1: 1-11.

6. Barros JAC. Propaganda de medicamentos: atentado à saúde? São Paulo: Hucitec/Sobravime; 1995.

7. Tomasi E. et al. Condições de trabalho e automedicação em profissionais da rede básica de saúde da zona urbana de Pelotas, RS. Rev. Bras. Epidemiol. Rio Grande do Sul, 2007;10(1):66-74.

8. Loyola Filho Al., et al. Prevalência e fatores associados à automedicação: resultados do Projeto Bambui. Rev. Saúde Pública. São Paulo, 2002;36(1): 55-62.

9. Vitor RS. et al. Padrão de consumo de medicamentos sem prescrição médica na cidade de Porto Alegre, RS. Ciência \& Saúde Coletiva. 2008; 13: 737-743.

10. Paredes NP, Miasso Al, Tirapelli CR. Consumption of benzodiazepines without prescription among first-year nursing students at the University of Guayaquil, school of nursing, Ecuador. Rev. Latino-am Enfermagem. 2008; 16(1):634- 639.

11. Vieira FS. Possibilidades de contribuição do farmacêutico para a promoção da saúde. Ciênc. saúde coletiva. 2007; 12(1): 213-220.

12. CIM, Centro de Informação sobre Medicamentos. Avaliação de problemas de saúde leves e autolimitados. CIM Formando n 02 - Mai/Jun/Jul/Ago de 2007.
13. Zubioli A. O farmacêutico e a automedicação responsável. Pharmacia Brasileira - Set/Out 2000.

14. Barros ARR, Griep RH, Rotenberg L. Automedicação entre os trabalhadores de enfermagem de hospitais públicos. Ver Latinoam Enferm, 2009;17(6):15-22.

15. Loyola Filho Al, Uchoa E, Gurra HL, Firmo JOA, Lima-Costa MF. Prevalência e fatores associados à automedicação: resultados do projeto Bambuí. Revista de Saúde Pública,2002;36(1), 55-62.

16. Bush PJ, Osterweis M. Pathways to medicine use. J Health Soc Behav 1978;19(1):79-89.

17. Loyola Filho Al, Uchoa E, Firmo JOA, LimaCosta MF. Estudo de base populacional sobre o consumo de medicamentos entre idosos: Projeto Bambuí. Cadernos de Saúde Pública, Rio de Janeiro, março/abril, 2005; 21(2):545553.

18. Rozenfeld S. Prevalência, fatores associados de mal uso de medicamentos entre idosos: uma revisão. Cad Saúde Pública RJ 2003;19(3):17-24.

19. Sá MB, Barros JAC, Oliveira Sá MPB. Automedicação em idosos na cidade de Salgueiro - PE. Rev Bras Epidemiol, 2007;10(1):75-85.

20. Figueiras $A$, Caamaño $F$. Sociodemographic factors related to self-medication in Spain. Eur J Epidemiol 2000;16(2):19-26.

21. Leite SN, Vieira M, Veber AP. Drug utilization studies: a synthesis of articles published in Brazil and Latin America. Cienc saude coletiva, 2012;13(0):793-802.

22. Baggio MA, Formaggio FM. Self-medication: Showing self carelessness by nursing professionals. Rev Enferm Uerj, 2012;17(2):48.

23. Barros ARR, Griep RH, Rotenberg L. Automedicação entre os trabalhadores de enfermagem de hospitais públicos. Rev Latino-Am Enfermagem, 2009;17(6):15-22.

24. Silva LSF, Costa AMDD, Terra FS, Zanetti HHV, Costa RD, Costa MD. Self-medication made by undergraduate degree courses of a private university's health area in the Minas Gerais' southern. Odontol clin cient, 2012;10(1):57-63.

25. Bock L, Tarantino M. Atração: o brasileiro exagera nos remédios, consumindo-os sem consultar o médico e colocando sua saúde em risco. Isto é.,v.1671, 2001.

26. Lopes NM. Automedicação: algumas reflexões sociológicas. Sociologia, Problemas e Práticas, 2001;37(2):41-65. 
27. Instituto de Pesquisa e Pós-Graduação do Mercado Farmacêutico -ICTQ. Pesquisa sobre uso racional de medicamentos no Brasil. 2014.

28. Santos B. Este consumo que nos consome olhares sobre a sociedade de consumo atual. Porto, Campo das Letras, 2006.

29. Casagrande EF, Gomes EA, Lima LCB, Oliveira ME, Oliveira RN, Riani RLA, Oliveira TB, Pinheiro RO. Estudo da utilização de medicamentos pela população universitária do município de Vassouras (RJ). Infarma, 2004;16(5/6):86-88.

30. Martins ERC. As substâncias psicoativas e o trabalhador de enfermagem. [Dissertação]. Rio de Janeiro: Escola de Enfermagem Anna Nery/UFRJ, 2006 [cited 2015 Mar 20].

31. Paulo LG, Zanini AC. Automedicação no Brasil. Revista Ass Méd Brasil, São Paulo, 1997;34(2):69-75.

32. Peixoto JB. Automedicação no adulto. Universidade Fernando Pessoa, Ponte de Lima, 2008.

33. Marques FB. Medicamentos e Farmacêuticos. Lisboa, Campo da Comunicação. 2006.

34. Organização Mundial de Saúde. O passado e o futuro dos medicamentos, editorial, Revista a Saúde do Mundo, 1998.

35. Pin JG. O profissional de enfermagem e a dependência química por psicofármacos: uma questão de saúde do trabalhador. [Dissertação]. Rio de Janeiro (RJ): Escola de Enfermagem Anna Nery/UFRJ; 1999.

36. Ritu P. et al. An online exploratory study of self medication among pharmacy graduates in India. Int. J. Drug Dev. \& Res., 2011;3(4):200 - 207.

37. Ebrahim A, Balbisi EA, Ambizas EM. Selfprescribing of noncontrolled substances among pharmacists. Am J Health-Syst Pharm, Bethesda, 2005;62(1):08-11.

38. Schneider M, Gallacchi MB, Goehring C, Künzi B, Bovier PA. Personal use of medical care and drugs among Swiss primary care physicians. Swiss Med Wkly 2007;137(7):2161.

39. Vilarino, J.F., et al. Perfil da automedicação em Município do Sul do Brasil. Rev. Saúde Pública, Rio Grande do Sul, 1998;32(1):4349. 